\title{
Pulsed-field gel electrophoresis (PFGE) analysis of Listeria monocytogenes isolates from different sources and geographical origins and representative of the twelve serovars
}

\author{
Elsa Neves ${ }^{\mathrm{a}, \mathrm{b}}$, António Lourenço ${ }^{\mathrm{a}}$, Ana Carla Silva ${ }^{\mathrm{a}}$, Rute Coutinho ${ }^{\mathrm{a}}$, Luisa Brito ${ }^{\mathrm{a}, *}$ \\ ${ }^{a}$ Laboratório de Microbiologia, CBAA/DBEB, Instituto Superior de Agronomia, Technical University of Lisbon, Tapada da Ajuda, \\ 1349-017 Lisbon, Portugal \\ ${ }^{\mathrm{b}}$ Instituto Superior de Estudos Interculturais e Transdisciplinares (ISEIT), Campus Universitário de Almada, Instituto Piaget, \\ Quinta da Arreinela de Cima, 2800-305 Almada, Portugal
}

Received 7 July 2008

\begin{abstract}
Multiplex-PCR (MPCR) serogrouping and pulsed-field gel electrophoresis (PFGE) subtyping analysis are currently used by several public and private laboratories for the characterization of Listeria monocytogenes. In this study a set of $80 \mathrm{~L}$. monocytogenes isolates belonging to the twelve serovars was used to investigate (i) the typeability of the rare serovars, (ii) the ability of PFGE analysis with $A p a \mathrm{I}$ and $A s c \mathrm{I}$ to differentiate serovars within MPCR serogroups and (iii) the association of molecular types with the specific source or geographical origin of the isolates. With the exception of three isolates (rare serovars $4 \mathrm{a}$ and $4 \mathrm{c}$ ) that were not amenable to restriction with $A p a \mathrm{I}$, all the other analyzed isolates were subtyped by both enzymes. PFGE discriminated the 80 isolates into 62 combined ApaI and AscI PFGE patterns (pulsotypes), but could not differentiate serovars within MPCR serogroups, in which isolates from different serovars displaying the same pulsotype were found. Clustering analysis suggests that for some pulsotypes grouping according to Portuguese origin or source can be suggested. On the other hand, some L. monocytogenes clones are widely distributed. Two pulsotypes from Portuguese human isolates were identical to the ones displayed by human outbreak clones in the UK and in the USA and Switzerland, respectively, although they were not temporally matched.

Computer-assisted data analysis of large and diverse PFGE type databases will improve the correct interpretation of subtyping data in epidemiological studies and in tracing routes and sources of contamination in the food industry. (C) 2008 Elsevier $\mathrm{GmbH}$. All rights reserved.
\end{abstract}

Keywords: Listeria monocytogenes; Sources; Geographical origin; Genetic diversity; Serovars; Genetic lineages; PFGE; Multiplex-PCR (MPCR) serogrouping

\footnotetext{
*Corresponding author. Tel.: + $351213653240,+351213653435$; fax: +351213653238

E-mail address: lbrito@isa.utl.pt (L. Brito).
}

\section{Introduction}

Listeria monocytogenes is a foodborne facultative intracellular pathogenic bacterium responsible for listeriosis in humans and animals, particularly among immunocompromised individuals. Thirteen serovars (1/2a, 1/2b, 1/2c, 3a, 3b, 3c, 4a, 4ab, 4b, 4c, 4d, 4e, 7) 
have been reported for L. monocytogenes [5]. Some of these serovars have been recovered from patients and foods $(1 / 2 \mathrm{a}, 1 / 2 \mathrm{~b}, 1 / 2 \mathrm{c}$ and $4 \mathrm{~b})$ more frequently [14] than the others considered as rare serovars. Although serotyping [20] is less discriminatory than molecular typing methodologies, it has been used in epidemiological studies as a universal technique for the characterization of L. monocytogenes. Recently, different authors have proposed PCR based methods for serogrouping L. monocytogenes isolates [1,5,24].

The multiplex-PCR (MPCR) assay proposed by Doumith et al. [5] is based on the use of five sets of primers targeting group-specific genes that enable the clustering of $L$. monocytogenes strains into five phylogenetic groups (serogroups) correlated with the serovars (except serovar $4 \mathrm{ab}$ which is extremely rare) [5]. The set of primers targeting the prs gene (putative phosphoribosyl pyrophosphate synthetase) fragment (370 bp) is universal to all Listeria species. In addition to this fragment, strains from group $1(1 / 2 \mathrm{a}-3 \mathrm{a})$ generate one amplified fragment from gene lmo0737 (unknown function) (691 bp), strains from group 2 (1/2c-3c) produce two fragments from gene $\operatorname{lmo} 0737$ (691 bp) and gene $l m o 1118$ (unknown function) (906 bp), strains from group $3(1 / 2 b-3 b-7)$ amplify a fragment from ORF2819 (putative transcriptional regulator) (471 bp), and strains from group $4(4 \mathrm{~b}-4 \mathrm{~d}-4 \mathrm{e})$ generate two fragments from ORF2819 (471 bp) and from ORF2110 (putative secreted protein) (597 bp). However, the proposed MPCR assay does not distinguish $L$. monocytogenes serovar $1 / 2 \mathrm{a}$ from $3 \mathrm{a}, 1 / 2 \mathrm{c}$ from $3 \mathrm{c}, 1 / 2 \mathrm{~b}$ from $3 \mathrm{~b}$ and 7 , $4 \mathrm{~b}$ from $4 \mathrm{~d}$ and $4 \mathrm{e}$. Isolates from serovars $4 \mathrm{a}$ and $4 \mathrm{c}$ (grouped as $\mathrm{L}$ in the present work) and isolates from the other Listeria species are also undistinguishable by this approach.

In order to better differentiate $L$. monocytogenes isolates, different subtyping methods have been used $[10,22,23]$ although pulsed-field gel electrophoresis (PFGE) is the current gold standard method for molecular subtyping of most foodborne bacteria, including L. monocytogenes [8]. Results from different subtyping studies suggest that three genetic divisions or lineages correlated with the flagellar $(\mathrm{H})$ antigene type exist in L. monocytogenes [2,15,19,23]. Lineage I consists of strains (flagellar antigen types b and d) that are more likely to cause human disease than isolates classified into lineages II (antigen type a or c) and III (serovars 4a and 4c and some $4 \mathrm{~b}$ strains). However, clustering analysis based on these studies usually uses isolates from the more frequent serovars ( $1 / 2 \mathrm{a}, 1 / 2 \mathrm{~b}, 1 / 2 \mathrm{c}$ and $4 \mathrm{~b})$.

Eighty isolates collected in Portugal and out of Portugal from different sources (including humans, animals, food and environment), and belonging to twelve serovars were analyzed in this study. We used MPCR serogrouping and PFGE subtyping to investigate (i) the typeability of the rare serovars, (ii) the ability of PFGE to differentiate serovars of L. monocytogenes within MPCR serogroups and (iii) the association of molecular types with specific sources or geographical origin (from Portugal versus out of Portugal). To our knowledge, this is the first report on clustering analysis of PFGE patterns of a group of isolates representing the twelve serovars of $L$. monocytogenes.

\section{Materials and methods}

\section{Bacterial strains and growth media}

Eighty L. monocytogenes isolates (Fig. 1) including 13 culture collection strains, previously classified by slide agglutination serotyping, and belonging to the twelve serovars were characterized in this study. The strains were from food $(n=29)$, from human cases of listeriosis $(n=25)$, from animals $(n=4)$, from environment $(n=5)$ and from uncertain sources $(n=17)$ (Fig. 1). The geographical origin of the isolates was ascribed as from Portugal (P) or from out of Portugal (O), as it was in most of the cases the only information we had. Some of the L. monocytogenes strains were kindly provided by the Faculté de Médecine de Tours, Université FrançoisRabelais (L'U.F.R), France, Laboratoire des Listeria, Institut Pasteur (I.P.) Paris, France, Universidade do Algarve (UA), Portugal and Università degli studi di Udine (USU), Italy. The 13 reference strains were from Collection de l'Institut Pasteur (CIP), Colleción Española de Cultivos Tipo (CECT) and National Collection of Type Cultures (NCTC): CIP104794 ( = NCTC7973) and NCTC7973 (serovar 1/2a); CECT936 and NCTC10887 (serovar 1/2b); CECT911 ( = ATCC19113) (ATCC-American Type Culture Collection) and NCTC9862 (serovar 1/2c); NCTC5105 (serovar 3a); CECT937 and CIP78.35 (serovar 3b); CECT934 ( = ATCC19114) (serovar 4a); CECT 4032 (NCTC11994) and NCTC10527 (serovar 4b); CIP78.39 ( = ATCC19116) (serovar 4c).

All stains were kept on Tryptic Soy Broth (TSB, Difco, Detroit, USA) containing $15 \%$ glycerol and stored at $-80^{\circ} \mathrm{C}$. Before the assays, each $L$. monocytogenes selected isolate was streaked on TSA-YE (Oxoid, Hampshire, UK) plates, and incubated for $18 \mathrm{~h}$ at $37^{\circ} \mathrm{C}$. Queries of the PathogenTracker database (http://www. pathogentracker.net) which contains subtype information for 648 L. monocytogenes (as of 19/06/2008) from various sources were conducted to probe the distribution of 22 out of 45 Portuguese isolates.

\section{Multiplex-PCR (MPCR) serogrouping}

MPCR serogrouping was performed using as PCR templates lysates obtained from bacterial colonies, 

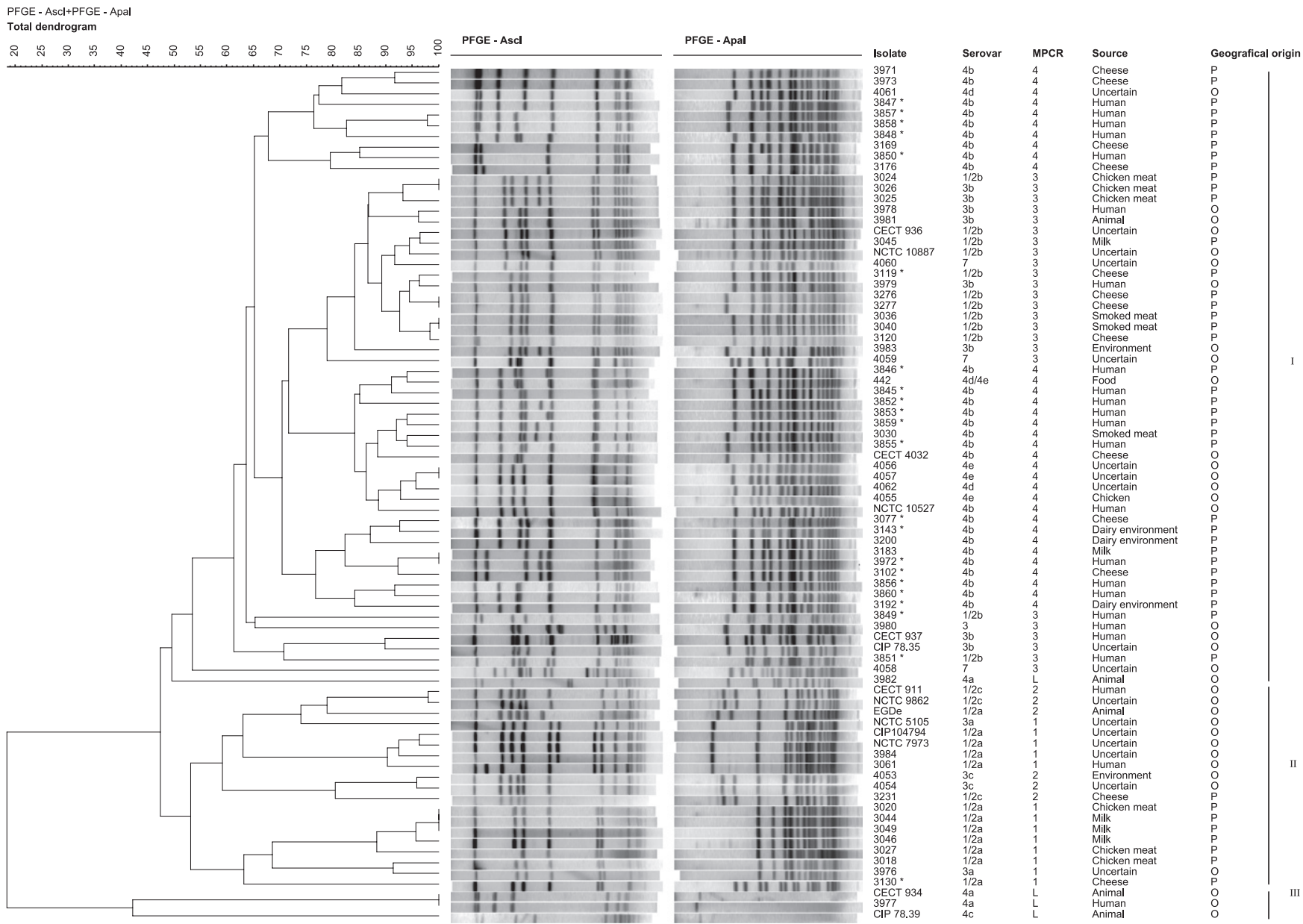

Fig. 1. Dendrogram (UPGMA clustering based on Dice correlation coefficient) of Listeria monocytogenes ApaI and AscI PFGE patterns for $80 \mathrm{~L}$. monocytogenes isolates. GelCompar version 4.5 was used with both a position tolerance and optimization of $1.50 \%$ for band comparison.

The reference of the isolates as well as their source, serovar and geographical origin (P-from Portugal, O-from out of Portugal) are indicated. Multiplex-PCR serogroup (MPCR) is indicated: 1 (serovars 1/2a-3a); 2 (serovars 1/2c-3c); 3 (serovars 1/2b-3b-7); 4 (serovars $4 \mathrm{~b}-4 \mathrm{~d}-4 \mathrm{e}$ ) and L (serovars $4 \mathrm{a}-4 \mathrm{c}$ ). The three genetic lineages of L. monocytogenes (I, II and III) are indicated. Strains from culture collections are identified by their original reference: CECT-Colleción Española de Cultivos Tipo; NCTC-National Collection of Type Cultures; CIP-Collection de l'Institut Pasteur. The patterns from the 22 isolates signed with an asterisk were searched in the PathogenTracker database.

according to Doumith et al. [5]. The five primer sets for target fragments from genes lmo0737, Imo1118, ORF2819, ORF2110 and prs were synthesized by MWG-Biotech AG Switzerland, according to the sequence data of Doumith et al. [4]. The resulting PCR products were resolved on $1.4 \%(\mathrm{w} / \mathrm{v})$ agarose gels in $1 \times$ TAE buffer, at $2.3 \mathrm{~V} / \mathrm{cm}$ for $60 \mathrm{~min}$. The gels were stained with ethidium bromide (Sigma) and analysed by using Bio-Rad Gel Doc $2000^{\mathrm{TM}}$ imaging system (Bio-Rad Laboratories, Segrate, Milan Italy).

\section{PFGE}

The $80 \mathrm{~L}$. monocytogenes isolates were characterized by DNA macrorestriction analysis using PFGE in accordance with the Pulse-Net standardized protocol [9]. Bacterial cultures were embedded in chromosomal grade agarose (SeaKem Gold Agarose, Cambrex, New Jersey, USA), lysed, washed and the DNA was in situ digested in separate reactions with $10 \mathrm{U}$ of $A s c \mathrm{I}$ (New England Biolabs, Massachusetts, USA) at $37^{\circ} \mathrm{C}$ and with $20 \mathrm{U}$ of ApaI (Roche Diagnostics, Mannheim, Germany) at $30^{\circ} \mathrm{C}$ overnight, respectively. The resolution of the generated DNA fragments was obtained with $1 \%$ SeaKem Gold Agarose gels in $0.5 \times$ Tris-Borate EDTA Buffer (Invitrogen, Paisley, UK) at $14{ }^{\circ} \mathrm{C}$ and $6 \mathrm{~V} / \mathrm{cm}$ with time ramped for $4-40 \mathrm{~s}$ over $22 \mathrm{~h}$ using a CHEF DR II System (BioRad, Hercules, USA). Gels were stained with ethidium bromide at a final concentration of $10 \mu \mathrm{g} / \mathrm{ml}$ (Sigma, St. Louis, USA) and pattern images were acquired in Bio-Rad Gel Doc $2000^{\mathrm{TM}}$ 
imaging system (Bio-Rad Laboratories, Milan, Italy). The TIF images were normalized by aligning the peaks of the size standard strain Salmonella enterica serovar Braenderup H9812 which was loaded on three lanes in each gel. This strain was kindly provided by Centre National de Référence des Listeria, Institut Pasteur, France.

\section{Data analysis}

PFGE patterns were analyzed and compared using the GelCompar II version 4.5 (Applied Maths, Kortrijk, Belgium). The levels of similarity were based on the Dice correlation coefficient. For cluster analysis of the patterns the unweighted-pair group matching algorithm (UPGMA) was used with a tolerance of $1.50 \%$ and optimization of $1.50 \%$ as recommended by Martin et al. [13]. The quality of the cluster analysis was verified by calculating the cophenetic correlation value $(\rho)$ for the dendrogram. Eight control strains of L. monocytogenes were used to determine the experimental variation between duplicate experiments. For both enzymes, the minimum level of repeatability of the macrorestriction conditions was calculated by running DNA samples from duplicated restrictions of the DNA of each strain. On the basis of the obtained results, a cut-off value of similarity was established for typing identical strains with identical outputs.

The Simpson's index of discrimination (SID) of PFGE, expressed as a percentage, was determined as described by Hunter and Gaston [12].

\section{Results and discussion}

MPCR and PFGE analysis are currently used by several public and private laboratories for serogrouping and subtyping L. monocytogenes. The first method constitutes a pratical alternative to slide agglutination serotyping and separates L. monocytogenes isolates belonging to the twelve serovars into five distinct serogroups. PFGE is considered the gold standard method for subtyping foodborne pathogens, because of its high discriminatory power and reproducibility. In this study, the combination of these methodologies with computer-assisted data analysis was applied to an isolate set representing considerable diversity (eighty isolates belonging to the twelve serovars representatives of the three genetic lineages of $L$. monocytogenes).

By using MPCR serogrouping, the 80 isolates were assigned to five groups (1-4 and L) (Fig. 1) in accordance with previous results from conventional serotyping. The exception was the strain EGDe (serovar $1 / 2 \mathrm{a})$ that showed a profile corresponding to MPCR serogroup $2(1 / 2 c-3 c)$ (Fig. 1) in agreement with a previous report of its unusual behaviour in this respect [5]. In accordance with this, the AscI and ApaI PFGE patterns of this strain were closer to the ones displayed by two culture collection strains from serovar 1/2c $(0.79$ similarity) than to the other patterns displayed by the serovar 1/2a isolates analysed (Fig. 1).

Strain 3980 given to us as serovar 3, displayed a multiplex pattern for group $3(1 / 2 b-3 b-7)$, suggesting to be a serovar $3 \mathrm{~b}$ isolate. This result was confirmed by the location of this isolate in the dendrogram (Fig. 1).

The results of multiplex-PCR of strains from the rare serovars $4 \mathrm{a}$ and $4 \mathrm{c}$ (group $L$ ) showed a negative influence of biomass excess in the cell lysate. In fact, in addition to the expected $370 \mathrm{bp}$ fragment, a faint band of about $471 \mathrm{bp}$ was present in the first amplification profiles of these strains (results not shown). This fact could lead into an incorrect assignment of these strains to multiplex group 3 (serovars $1 / 2 b-3 b-7$ ). Nightingale et al. [17] also reported the misclassification of strains from serovars $4 \mathrm{a}$ and $4 \mathrm{c}$ in multiplex group 3 . In the last trials using a lysate with less biomass, this faint band disappeared pointing out the need to work in stringent compliance with the protocol.

PFGE of the DNAs of the 80 isolates digested with AscI showed 7-16 fragments ranging from approximately 30 to $1130 \mathrm{~kb}$ in size, while $11-16$ fragments of $30-510 \mathrm{~kb}$ were obtained following digestion using ApaI. The genomic DNA from two out of the three strains from serovar 4a (strain CECT 934 and isolate 3977) and strain CIP 78.39 (serovar 4c) were not amenable to digestion with $A p a \mathrm{I}$ (Fig. 1). Similar results were previously reported by Brosch et al. [2] for strain ATCC19116 (serovar 4c) equivalent to our strain CIP78.39.

The relationships among $L$. monocytogenes strains based on their combined ApaI and AscI PFGE profiles are shown in the dendrogram displayed in Fig. 1, and are supported by a cophenetic correlation coefficient $(\rho)$ equal to 0.92 . This value explained the goodness of fit of the clustering to the original data [21] and usually ranges between 0.60 and 0.95 and values higher than 0.80 are considered reasonable [18].

Based on data obtained from the duplicate experiments, a cut-off level of 0.95 similarity was established for the definition of a PFGE pattern. PFGE of AscI macrorestriction fragments differentiated the 80 isolates into 33 different patterns. The PFGE of ApaI macrorestriction fragments of the 77 typeable isolates produced 44 different patterns. A higher SID value was achieved with $A p a \mathrm{I}$ (0.91) compared to $A s c \mathrm{I}(0.85)$. The combination of patterns produced by both enzymes yielded 62 combined $A p a \mathrm{I}$ and AscI PFGE types (pulsotypes) increasing the discriminatory power up to 0.97 (Fig. 1).

At about 0.42 similarity level L. monocytogenes isolates could be divided into two major clusters. The 
smaller cluster corresponded to lineage III (three isolates from serovars $4 \mathrm{a}$ and $4 \mathrm{c}$ typed only by $A s c \mathrm{I}$ ) well separated from the bigger cluster that gathered at 0.50 of similarity two subgroups. In one of these subgroups the 19 isolates from serovars $1 / 2 \mathrm{a}, 1 / 2 \mathrm{c}, 3 \mathrm{a}$ and $3 \mathrm{c}$ (lineage II) were clustered. The other subgroup gathered the 58 isolates from serovars $1 / 2 \mathrm{~b}, 3 \mathrm{~b}, 4 \mathrm{~b}, 4 \mathrm{~d}, 4 \mathrm{e}$ and 7 (lineage I). These results are in agreement with Doumith et al. [4] that used a DNA macroarray-based subtyping method to divide the three lineages of $L$. monocytogenes into five phylogenetic groups, each correlated with serovars. In fact, clustering of the isolates according to their pulsotypes is in accordance with MPCR serogroups.

Nevertheless, PFGE subtyping could not differentiate serovars within MPCR serogroups. Moreover, within serogroups identical pulsotypes were shared by isolates belonging to different serovars: serogroup 3 (serovars $1 / 2 \mathrm{~b}$ and $3 \mathrm{~b}$, isolates 3024 and 3026) and serogroup 4 (serovars 4e and 4d, isolates 4056, 4057 and 4062) (Fig. 1). Interestingly, two isolates belonging to MPCR serogroup 4 (serovars $4 \mathrm{~b}-4 \mathrm{~d}-4 \mathrm{e}$ ) had significantly different levels of pathogenicity (evaluated both in vivo and in vitro) [16]: human isolate 3846 (4b) was significantly more virulent than food isolate $442(4 \mathrm{~d} / 4 \mathrm{e})$ although their pulsotypes had about $0.94 \%$ similarity.

For some isolates, similarity clustering analysis of PFGE patterns suggested association between molecular types and specific sources or Portuguese origin, in accordance with previous reports [3,7]. At 0.53 similarity level, 19 isolates from lineage II were clustered in two subgroups (Fig. 1). In one of these subgroups seven out of eight isolates were from Portugal and collected from food (chicken meat and milk). There was no evidence that the isolates from milk were related or not and there was no acknowledged association between the chicken meat and the milk. In the other subgroup, the eleven isolates were mainly from out of Portugal and from diverse sources.

At 0.73 similarity level (Fig. 1) the isolates from lineage I were grouped in three major subgroups. One of these subgroups gathered nine L. monocytogenes (serogroup 4) Portuguese isolates from milk, cheese, dairy environment and from human cases of listeriosis. Two of these isolates (3183 and 3972) collected in 2002 from raw milk, and in 2004 from placenta, respectively, have identical pulsotypes. In this cluster, there are isolates from milk and cheese (3077 and 3143) persistently collected in two different cheese dairies.

At the same similarity level, the two other major clusters with 18 and 14 isolates (serogroups 3 and 4, respectively) were diverse in what geographical origin (from Portugal and from out of Portugal) and source (type of food, humans, environment and animal) are concerned. The wide distribution of some of these pulsotypes was confirmed by querying the PathogenTracker database for 22 selected isolates. Isolate 3845 collected in 2000 in Portugal from an infected person shared the same pulsotype (designated in Fugett et al. [6] by pulsotype 7) with 17 isolates in PT database: bovine (2), human (5), water (4), food (2), caprine (2), enviroment (2). Among this group were isolates involved in two outbreaks related to cheese consumption, in Los Angeles (LA) and in USA (1985) and Switzerland (1983-1987) [6]. Portuguese isolates 3857 and 3858 collected from two infected persons in 1997 and 1998, respectively, had a pulsotype indistinguishable from pulsotype 20 displayed by isolates involved in a human epidemic case related to pâté consumption in 1988-1990, in the UK [6].

Computer-assisted data analysis of PFGE data simplifies the processing of a large number of samples while allowing data sharing if a stringent compliance to standard protocols is achieved. This standardization includes not only a rigorous accomplishment of the Pulse-Net standardized protocol by Graves and Swaminathan [9] but also standardization of procedures in computer-assisted data analysis for pattern normalization and transfers of information intra and inter laboratories.

PulseNet Europe [13] recommends settings of both optimization and position tolerance at $1.5 \%$ for band comparison in accordance with Pulsenet USA. Although complying with these settings, strains visually indistinguishable may still be considered different according to the clustering analysis [11]. Aiming to minimize this, the determination of the experimental variation between duplicate experiments was evaluated in this work, allowing an establishment of a cut-off value of similarity of 0.95 for typing identical PFGE patterns. In spite of this, visual PFGE matches were found different by computer analysis (e.g. serovar 4b isolates 3102 (cheese), 3183 (milk) and 3972 (human)). Owing to this, a causal relationship between food and human isolates cannot be deduced without an additional subtyping method, in preference amenable to automation and based on DNA sequence. Nevertheless, to improve correct interpretation of subtyping data, the availability of a large and diverse PFGE type database is needed.

To our knowledge, this is the first report on clustering analysis of PFGE patterns of a diversity set of strains from twelve out of the thirteen serovars representative of all three genetically different $L$. monocytogenes lineages.

\section{Acknowledgments}

The authors thank Dr. Alban Le Monnier (I.P.), Dr. Laurent Mereghetti (L'U.F.R.), Dr. Leonor Faleiro (UA) and Dr. Luca Cocolin (USU) for providing some of the L. monocytogenes isolates and reference strains 
used in this study. The authors thank Dr. Martin Wiedmann and Yesim Soyer for queries to the PathogenTracker database.

The financial support of Fundação para a Ciência e Tecnologia (FCT) (Projects POCI/SAU-ESP/56243/2004 and PPCDT/SAU-ESP/56243/2004, Project REEQ/348/ AGR/2005 and $\mathrm{PhD}$ grant SFRH/BD/17914/2004 of Elsa Neves) is gratefully acknowledged.

\section{References}

[1] M.K. Borucki, D.R. Call, Listeria monocytogenes serotype identification by PCR, J. Clin. Microbiol. 41 (2003) 5537-5540.

[2] R. Brosch, J. Chen, J.B. Luchansky, Pulsed-field fingerprinting of Listeriae: identification of genomic divisions for Listeria monocytogenes and their correlation with serovars, Appl. Environ. Microbiol. 60 (1994) 2584-2592.

[3] L. Cocolin, S. Stella, R. Nappi, E. Bozzetta, C. Cantoni, G. Comi, Analysis of PCR-based methods for characterization of Listeria monocytogenes strains isolated from different sources, Int. J. Food Microbiol. 103 (2005) 167-178.

[4] M. Doumith, C. Cazalet, N. Simoes, L. Frangeul, C. Jacquet, F. Kunst, P. Martin, P. Cossart, P. Glaser, C. Buchrieser, New aspects regarding evolution and virulence of Listeria monocytogenes revealed by comparative genomics and DNA arrays, Infect. Immun. 72 (2004) 1072-1083.

[5] M. Doumith, C. Buchrieser, P. Glaser, C. Jacquet, P. Martin, Differentiation of the major Listeria monocytogenes serovars by multiplex PCR, J. Clin. Microbiol. 42 (2004) 3819-3822.

[6] E. Fugett, E. Fortes, C. Nnoka, M. Wiedmann, International Life Sciences Institute North America Listeria monocytogenes strain collection: development of standard Listeria monocytogenes strain sets for research and validation studies, J. Food Prot. 69 (2006) 2929-2938.

[7] E.B. Fugett, D. Schoonmaker-Bopp, N.B. Dumas, J. Corby, M. Wiedmann, Pulsed-Field Gel Electrophoresis (PFGE) analysis of temporally matched Listeria monocytogenes isolates from human clinical cases, foods, ruminant farms, and urban and natural environments reveals source-associated as well as widely distributed PFGE types, J. Clin. Microbiol. 45 (2007) 865-873.

[8] P. Gerner-Smidt, K. Hise, J. Kincaid, S. Hunter, S. Rolando, E. Hyytia-Trees, E.M. Ribot, B. Swaminathan, PulseNet USA: a five-year update, Foodborne Pathog. Dis. 3 (2006) 9-19.

[9] L.M. Graves, B. Swaminathan, PulseNet standardized protocol for subtyping Listeria monocytogenes by macrorestriction and pulsed-field gel electrophoresis, Int. J. Food Microbiol. 65 (2001) 55-62.

[10] M.M. Guerra, F. Bernardo, J. McLauchlin, Amplified fragment length polymorphism (AFLP) analysis of Listeria monocytogenes, Syst. Appl. Microbiol. 25 (2002) 456-461.
[11] T.M. Hamdi, M. Naïm, P. Martim, C. Jacquet, Identification and molecular characterization of Listeria monocytogenes isolated in raw milk in the region of Algiers (Algeria), Int. J. Food Microbiol. 116 (2007) 190-193.

[12] P.R. Hunter, M.A. Gaston, Numerical index of the discriminatory ability of typing schemes: an application of Simpson's index of diversity, J. Clin. Microbiol. 26 (1988) 2465-2466.

[13] P. Martin, C. Jacquet, V. Goulet, V. Vaillant, H. De Valk, Pulse-Field Gel Electrophoresis of Listeria monocytogenes strains: the PulseNet Europe feasibility study, Foodborne Pathog. Dis. 3 (2006) 3003-3308.

[14] J. McLauchlin, R.T. Mitchell, W.J. Smerdon, K. Jewell, Listeria monocytogenes and listeriosis: a review of hazard characterization for use in microbiological risk assessment of foods, Int. J. Food Microbiol. 92 (2004) 15-33.

[15] C.A. Nadon, D.L. Woodward, C. Young, F.G. Rodgers, M. Wiedmann, Correlations between molecular subtyping and serotyping of Listeria monocytogenes, J. Clin. Microbiol. 39 (2001) 2704-2707.

[16] E. Neves, A.C. Silva, S.M. Roche, P. Velge, L. Brito, Virulence of Listeria monocytogenes isolated from cheese, dairy environment and from other foods and clinical cases, J. Med. Microbiol. 57 (2008) 411-415.

[17] K. Nightingale, L. Bovell, A. Grajczyk, M. Wiedmann, Combined $\operatorname{sig} B$ allelic typing and multiplex PCR provide improved discriminatory power and reliability for Listeria monocytogenes molecular serotyping, J. Microbiol. Methods 68 (2007) 52-59.

[18] F. Priest, B. Austin, Modern Bacterial Taxonomy, second ed., Chapman and Hall, London, 1993.

[19] O.F. Rasmussen, P. Skouboe, L. Dons, L. Rossen, J.E. Olsen, Listeria monocytogenes exists in at least three evolutionary lines: evidence from flagellin, invasive associated protein and listeriolysin $\mathrm{O}$ genes, Microbiology 141 (1995) 2053-2061.

[20] H.P.R. Seeliger, K. Höhne, Serotyping of Listeria monocytogenes and related species, in: T. Bergan, J. Norris (Eds.), Methods in Microbiology, Academic Press, New York, 1979, pp. 33-48.

[21] P.H.A. Sneath, R.R. Sokal, Numerical Taxonomy-The Principles and Practice of Numerical Classification, W. H. Freeman, San Francisco, 1973.

[22] K. Wernars, P. Boerlin, A. Audurier, E.G. Russell, G.D. Curtis, L. Herman, N. van der Mee-Marquet, The WHO multicenter study on Listeria monocytogenes sub-typing: random amplification of polymorphic DNA (RAPD), Int. J. Food Microbiol. 32 (1996) 325-341.

[23] M. Wiedmann, J.L. Bruce, C. Keating, A.E. Johnson, P.L. McDonough, A. Batt, Ribotypes and virulence gene polymorphisms suggest three distinct Listeria monocytogenes lineages with differences in pathogenic potential, Infect. Immun. 65 (1997) 2707-2716.

[24] W. Zhang, S.J. Knabel, Multiplex PCR assay simplifies serotyping and sequence typing of Listeria monocytogenes associated with human outbreaks, J. Food Prot. 68 (2005) 1907-1910. 